\title{
Reordering an existing queue
}

\author{
Youngsub Chun ${ }^{1}$. Manipushpak Mitra ${ }^{2}$. \\ Suresh Mutuswami ${ }^{3}$
}

Received: 22 March 2016 / Accepted: 5 April 2017 / Published online: 18 April 2017 (C) The Author(s) 2017. This article is an open access publication

\begin{abstract}
We consider the mechanism design problem of reordering an existing queue when waiting costs are unknown and processing times for jobs are identical. In contrast to the corresponding problem without an initial queue, budget balance (BB), outcome efficiency (OE), strategyproofness (SP), and individual rationality (IR) are incompatible. We investigate the implications of dropping BB and OE. Dropping BB yields no mechanism which never runs a budget deficit but allowing for a bound on the budget deficit yields positive results. Dropping OE yields only fixed-price trading or constant mechanisms when there are two agents but there are additional mechanisms when there are more agents. We identify a mechanism which is not a fixed-price trading mechanism and characterize its maximal level of 'queue inefficiency'.
\end{abstract}

We thank two anonymous referees and an associate editor for their detailed comments, Arunava Sen for the title and the Indian Statistical Institute, Kolkata for its hospitality. Chun's work was supported by the National Research Foundation of Korea Grant funded by the Korean Government (NRF-2016S1A3A2924944) and the Center for Distributive Justice, Seoul National University.

Suresh Mutuswami sm403@le.ac.uk

Youngsub Chun ychun@snu.ac.kr

Manipushpak Mitra mmitra@isical.ac.in

1 Department of Economics, Seoul National University, Seoul, South Korea

2 Economic Research Unit, Indian Statistical Institute, Kolkata, India

3 School of Business, Economics Division, University of Leicester, Leicester, UK 


\section{Introduction}

Queueing problems have been analyzed from different points of view [see Chun (2006a, b), Chun et al. (2014a, b), Gershkov and Schweinzer (2010), Kayi and Ramaekers (2010), Maniquet (2003), Mitra (2001), Mitra and Mutuswami (2011) among others]. With the exception of Curiel et al. (1989) and Gershkov and Schweinzer (2010), the papers have assumed that there is no initial order of the agents. However, many queueing situations involve an initial order. In such cases, agents are usually served according to the first-come, first-served protocol. While this rule is simple to implement, it is not desirable when waiting in a queue is costly for agents. The fact that trading of queue positions is allowed in some cases points to the desirability of reordering the initial queue. ${ }^{1}$

In this paper, we address the problem of reordering an existing queue from a mechanism design viewpoint. In our setup, agents have quasi-linear preferences and waiting costs are linear in time. We are interested in mechanisms which are budget balanced (the sum of the transfers to agents is zero), outcome efficient (the selected queue minimizes aggregate waiting costs) and strategyproof (no agent can benefit strictly by reporting untruthfully).

When there is no initial order, Chun et al. (2016), Mitra (2001), Kayi and Ramaekers (2010) and Suijs (1996) show the existence of "first-best" mechanisms satisfying all three properties. Hence, our problem is non-trivial only if the initial queue imposes additional requirements. One natural requirement is individual rationality. This requires that any reordering must give an agent at least the utility she would have obtained in the first-come, first-served protocol. Not only is this requirement natural, it is present in some practical situations too. ${ }^{2}$

Unfortunately, the above four properties are incompatible. ${ }^{3}$ We therefore examine the consequences of dropping budget balance and outcome efficiency, one at a time. We retain individual rationality for two reasons. Firstly, we know that if we drop individual rationality, then there are mechanisms satisfying the remaining three properties. ${ }^{4}$ Secondly, in our context, it makes sense to think that an agent will not agree to move to a different queue position if this leaves her strictly worse-off. We also retain strategyproofness as replacing it with a weaker notion like Bayesian incentive compatibility has been done by Gershkov and Schweinzer (2010).

Dropping budget balance gives us a sub-class of the family of the Vickrey-ClarkeGroves (VCG) mechanisms. All mechanisms in this sub-class run a budget deficit at some profile. Therefore, we have an impossibility result even if we weaken budget balance to no budget deficit. However, allowing for an upper bound on the budget

\footnotetext{
1 Gershkov and Schweinzer (2010) provide two examples: trading landing slots at some U.S. airports [see Schummer and Abizada (2016), Schummer and Vohra (2013)] and trading queue positions in the British National Health Service system.

2 Gershkov and Schweinzer (2010) note that patients have the right to reject the offered payment for switching queue positions in the British National Health Service system.

3 See Remark 2.9 and Theorem 3.7.

4 Instead of dropping individual rationality, we can replace it with a condition like identical preferences lower bound. We discuss this more in Remark 2.12.
} 
deficit gives a possibility result. A natural bound is the amount of cost saving (in terms of aggregate waiting cost) obtained by moving to the outcome efficient queue. We show that the pivotal mechanism and the minimum deficit VCG mechanism satisfy this upper bound.

Next, we drop outcome efficiency. For the case of two agents, we show that the only mechanisms satisfying budget balance, strategyproofness and individual rationality are either constant or fixed-price trading mechanisms. The former always selects the initial queue and assigns zero transfers for all agents. In a fixed-price trading mechanism, a price $p$ is chosen a priori. The two agents exchange positions if they both desire it, and the one moving forward pays $p$ to the one moving back. Neither type of mechanism is outcome efficient. ${ }^{5}$

With more than two agents, fixed-price and constant mechanisms are not the only ones satisfying budget balance, strategyproofness and individual rationality. Characterizing the set of mechanisms satisfying these three axioms is difficult because of our domain restriction. ${ }^{6}$ Hence, we simply identify a mechanism which is neither a fixed-price nor a constant mechanism. We also put a bound on its level of 'queue inefficiency'. This measure of inefficiency of a queue is based on the distance between the agent's queue position in the reordered queue and the agent's queue position in the outcome efficient queue.

The particular mechanism that we identify is the median waiting cost exchange mechanism. When the outcome efficient queue is unique, this mechanism works as follows for an odd number of $n$ agents. ${ }^{7}$ We first fix an order on all $n(n-1) / 2$ pairs of distinct agents $\{i, j\}$. Given a profile of waiting costs, we select the median waiting cost as the trading price. Moving in the fixed order, agents are allowed to trade their queue positions at the median price provided they both benefit strictly from the trade. ${ }^{8}$ It can be shown that median waiting cost exchange mechanism is at most $(n-1) / 2$-queue inefficient meaning that at any profile where the outcome efficient queue is unique, no agent is more than $(n-1) / 2$ positions away from where the agent would be in the outcome efficient queue.

We end the introduction by noting the differences between our work and the closest related work, Gershkov and Schweinzer (2010). Firstly, they look at Bayesian incentive compatible mechanisms. In this regard, strategyproof mechanisms offer the advantage that their specification is not dependent on the particular prior chosen by the mechanism designer. Secondly, Gershkov and Schweinzer (2010) are concerned with the distribution of property rights (over queue positions) for which the efficient queue correspondence can be implemented. On the other hand, we fix the property

\footnotetext{
5 Similar results obtain in other contexts also. See Barberà and Jackson (1995) and Hagerty and Rogerson (1987), for example.

${ }^{6}$ Specifically, we assume quasi-linear preferences. See Goswami et al. (2014) for a discussion of the difficulties that arise in characterizing strategyproof social choice functions in quasi-linear exchange economies even in the presence of Pareto efficiency.

${ }^{7}$ If $n$ is even, the mechanism can be adjusted appropriately. See Sect. 4.3.

8 To be precise, a trade involves the two agents exchanging their queue positions. The agent moving forward pays an amount equal to the median waiting costs times the number of places she moves forward. The agent moving back receives this amount.
} 
rights regime and examine the consequences of dropping budget balance and outcome efficiency, one at a time.

\section{The model}

Let $N=\{1, \ldots, n\}, n \geq 2$, be the set of agents. Each agent has one job to process. The processing time for all jobs is the same and without loss of generality, normalized to one. There is a server which can process only one job at a time.

A queue is an onto function $\sigma: N \rightarrow\{1, \ldots, n\}$ denoting the order in which agents are served. The set of all possible queues is denoted $\Sigma(N)$. There is an initial qиеие $\sigma^{0}$ which determines the order in which jobs will be processed if no reordering is done.

Given a queue $\sigma$ and an agent $i$, we denote her position in the queue by $\sigma_{i}$. The set of her predecessors is $P_{i}(\sigma)=\left\{j \in N \mid \sigma_{j}<\sigma_{i}\right\}$ and the set of followers is $F_{i}(\sigma)=\left\{j \in N \mid \sigma_{j}>\sigma_{i}\right\}$. The agent incurs a cost of waiting in the queue, given by $\left(\sigma_{i}-1\right) \theta_{i}$ where $\theta_{i} \in \mathbb{R}_{+}$is the waiting cost per unit of time. The value of $\theta_{i}$ is known only to agent $i$.

Agents have quasi-linear preferences given by $u_{i}\left(\sigma_{i}, t_{i} ; \theta_{i}\right)=-\left(\sigma_{i}-1\right) \theta_{i}+t_{i}$ where $t_{i}$ is the monetary transfer to agent $i .{ }^{9}$ In what follows, let $U_{i}\left(\sigma_{i}, t_{i} ; \theta_{i}\right)=$ $u_{i}\left(\sigma_{i}, t_{i} ; \theta_{i}\right)+\left(\sigma_{i}^{0}-1\right) \theta_{i}=\left(\sigma_{i}^{0}-\sigma_{i}\right) \theta_{i}+t_{i}$ denote the net utility gain of agent $i$ when she receives the allocation $\left(\sigma_{i}, t_{i}\right)$.

A profile $\theta \equiv\left(\theta_{1}, \ldots, \theta_{n}\right) \in \mathbb{R}_{+}^{n}$ is a vector of the waiting costs of all agents. The profile $\theta_{S}=\left(\theta_{i}\right)_{i \in S}$ is the vector of waiting costs of agents in the coalition $S$. The profiles $\theta$ and $\theta^{\prime}$ are $S$-variants if $\theta_{k}=\theta_{k}^{\prime}$ for all $k \notin S$. When $S=\{i\}$, we abuse notation slightly by using the term $i$-variant instead of $\{i\}$-variant.

A mechanism $\mu=(\sigma, t)$ associates with each profile $\theta$, a tuple $\mu(\theta) \equiv(\sigma(\theta), t(\theta))$ where $\sigma(\theta)$ is the reordered queue and $t(\theta)$ is the vector of transfers to the agents. Agent $i$ 's utility when her true waiting $\cos t$ is $\theta_{i}$ and the profile $\theta^{\prime}$ is reported to the mechanism is $u_{i}\left(\sigma_{i}\left(\theta^{\prime}\right), t_{i}\left(\theta^{\prime}\right) ; \theta_{i}\right) \equiv-\left(\sigma_{i}\left(\theta^{\prime}\right)-1\right) \theta_{i}+t_{i}\left(\theta^{\prime}\right)$.

\subsection{Axioms}

We discuss here the properties of mechanisms that are of interest. The first property requires that the mechanism not generate a surplus or deficit at every profile.

Definition 2.1 A mechanism $\mu=(\sigma, t)$ is budget balanced (BB) if for all profiles $\theta$, $\sum_{i=1}^{n} t_{i}(\theta)=0$.

As we will show, BB is incompatible with the other properties. We thus examine weaker variants, two of which are defined now. Feasibility requires that the mechanism never generate a deficit but allows it to generate a surplus. Weak Feasibility requires that the net transfers not exceed the saving in waiting cost achieved by the mechanism.

\footnotetext{
9 The status quo in this situation is for the jobs to be processed according to the initial queue with no transfers. In this case, agent $i$ 's utility is $-\left(\sigma_{i}^{0}-1\right) \theta_{i}$.
} 
Definition 2.2 A mechanism $\mu=(\sigma, t)$ is feasible (F) if for all profiles $\theta$, $\sum_{i=1}^{n} t_{i}(\theta) \leq 0$. It is weakly feasible (WF) if for all profiles $\theta, \sum_{i=1}^{n} t_{i}(\theta) \leq$ $\sum_{i=1}^{n}\left(\sigma_{i}^{0}-\sigma_{i}(\theta)\right) \theta_{i}$.

The next property requires that at all profiles, the selected queue should minimize the aggregate waiting cost. Formally, a queue $\sigma$ is efficient for the profile $\theta$ if $\sigma=$ $\operatorname{argmin}_{\sigma^{\prime} \in \Sigma(N)} \sum_{i \in N}\left(\sigma_{i}^{\prime}-1\right) \theta_{i}$. Let $E(\theta)$ be the set of all efficient queues at the profile $\theta$.

Definition 2.3 A mechanism $\mu=(\sigma, t)$ is outcome efficient (OE) if for all profiles $\theta, \sigma(\theta) \in E(\theta)$.

Remark 2.4 Outcome efficiency implies that agents are served in the non-increasing order of their waiting costs. Such a queue is not unique when two or more agents have the same waiting cost. Since our definition of a mechanism selects a unique queue at every profile, this axiom implicitly assumes the existence of a tie-breaking rule which selects an efficient queue whenever there is more than one such queue. We assume that there is a linear order of the agents according to which ties are broken. The same order is also used when we have to deal with subsets of agents.

Strategyproofness requires that truth-telling be a dominant strategy for all agents. This is a desirable property because it ensures that no agent can strictly benefit by misrepresenting her waiting cost no matter what she believes other agents to be doing. A stronger requirement is that the mechanism not be vulnerable to manipulation by groups. Two different variants of group strategyproofness can be defined. Weak group strategyproofness requires that there not exist a deviation for a coalition making all deviating members strictly better-off. Strong group strategyproofness requires that there not exist a deviation for a coalition making all deviating members weakly betteroff and at least one member strictly better-off.

Definition 2.5 A mechanism $\mu=(\sigma, t)$ is strategyproof (SP) if for all $i$-variants $\theta$ and $\theta^{\prime}, u_{i}\left(\sigma_{i}(\theta), t_{i}(\theta) ; \theta_{i}\right) \geq u_{i}\left(\sigma_{i}\left(\theta^{\prime}\right), t_{i}\left(\theta^{\prime}\right) ; \theta_{i}\right)$.

Definition 2.6 A mechanism $\mu=(\sigma, t)$ is weak group strategyproof if there does not exist $S \subset N$ and $S$-variants $\theta, \theta^{\prime}$ such that $u_{i}\left(\sigma_{i}\left(\theta^{\prime}\right), t_{i}\left(\theta^{\prime}\right) ; \theta_{i}\right)>u_{i}\left(\sigma_{i}(\theta), t_{i}(\theta) ; \theta_{i}\right)$ for all $i \in S$. It is strong group strategyproof if for all $S \subset N$ and all $S$-variants $\theta, \theta^{\prime}$, $u_{i}\left(\sigma_{i}\left(\theta^{\prime}\right), t_{i}\left(\theta^{\prime}\right) ; \theta_{i}\right) \geq u_{i}\left(\sigma_{i}(\theta), t_{i}(\theta) ; \theta_{i}\right)$ for all $i \in S$ implies $u_{i}\left(\sigma_{i}\left(\theta^{\prime}\right), t_{i}\left(\theta^{\prime}\right) ; \theta\right)=$ $u_{i}\left(\sigma_{i}(\theta), t_{i}(\theta) ; \theta_{i}\right)$ for all $i \in S$.

Finally, individual rationality requires that each agent's utility in the reordered queue be at least as large as the utility she would get if the jobs were processed according to the initial queue and no transfers are given. If a mechanism does not satisfy this property, then agents may not agree to trade their positions.

Definition 2.7 A mechanism $\mu=(\sigma, t)$ is individually rational (IR) if for all $\theta$ and all $i \in N, u_{i}\left(\sigma_{i}(\theta), t_{i}(\theta) ; \theta_{i}\right) \geq-\left(\sigma_{i}^{0}-1\right) \theta_{i}$.

Remark 2.8 We can alternatively specify IR as requiring for all $\theta$ and all $i \in N$, $U_{i}\left(\sigma_{i}(\theta), t_{i}(\theta) ; \theta_{i}\right) \geq 0$. 
Remark 2.9 Unfortunately, BB, OE, SP and IR are incompatible. We defer a proof of this result since we prove a stronger result in Theorem 3.7.

\subsection{Notation}

We introduce here three different measures of cost saving from reordering the initial queue. These will be useful in presenting the results that follow. The first measure is the maximal cost saving defined as the cost saving that can be achieved by reordering the initial queue into an efficient queue.

$$
\forall \theta \in \mathbb{R}_{+}^{n}: \quad B(\theta)=\max _{\sigma \in \Sigma(N)} \sum_{j \in N}\left(\sigma_{j}^{0}-\sigma_{j}\right) \theta_{j} .
$$

Next, suppose we fix the waiting costs of all agents in $N \backslash\{i\}$ and vary $i$ 's waiting cost to find the smallest cost saving from efficient reordering. We thus define the $i$-minimal cost saving as

$$
\forall \theta_{N \backslash\{i\}} \in \mathbb{R}_{+}^{n-1}: \quad B_{i}^{*}\left(\theta_{N \backslash\{i\}}\right)=\min _{\theta_{i}^{\prime} \in \mathbb{R}_{+}} B\left(\theta_{i}^{\prime}, \theta_{N \backslash\{i\}}\right) .
$$

Observe that $B\left(\theta_{i}^{\prime}, \theta_{N \backslash\{i\}}\right)$ is minimized when $\theta_{i}^{\prime}$ is such that agent $i$ 's position in the efficient queue (for the profile $\left(\theta_{i}^{\prime}, \theta_{N \backslash\{i\}}\right)$ ) is the same as her position in the initial queue. This is seen easily. Imagine that we keep agent $i$ 's position fixed and carry out efficient reordering with respect to the agents in $N \backslash\{i\}$. If agent $i$ 's initial queue position is not optimal, then further gains are possible by moving her to the optimal position. Hence, the minimal gain is when agent $i$ 's position in the efficient queue is the same as her initial queue position.

Finally, suppose that agent $i$ leaves the initial queue $\sigma^{0}$. Let everyone behind agent $i$ move up a place, so the initial queue is now

$$
\sigma_{j}^{0,-i}= \begin{cases}\sigma_{j}^{0} & \text { if } \sigma_{j}^{0}<\sigma_{i}^{0} \\ \sigma_{j}^{0}-1 & \text { if } \sigma_{j}^{0}>\sigma_{i}^{0}\end{cases}
$$

Efficient reordering of this queue gives us the third notion of cost saving, the maximal cost saving in the absence of agent $i$ :

$$
\forall \theta_{N \backslash\{i\}} \in \mathbb{R}_{+}^{n-1}: \quad B_{-i}^{*}\left(\theta_{N \backslash\{i\}}\right)=\max _{\sigma \in \Sigma(N \backslash\{i\})} \sum_{j \in N \backslash\{i\}}\left(\sigma_{j}^{0,-i}-\sigma_{j}\right) \theta_{j}
$$

\subsection{VCG mechanisms}

With quasi-linear preferences, it is known that the Vickrey-Clarke-Groves mechanisms (or VCG mechanisms) satisfy OE and SP [see Clarke (1971), Groves (1973), Vickrey (1961)]. In a classic paper, Holmström (1979) shows that the VCG mechanisms are the only ones satisfying OE and SP on convex domains. Since the domain of preferences 
is $\mathbb{R}_{+}^{n}$ in our context, it follows that the VCG mechanisms are the only ones satisfying $\mathrm{OE}$ and SP.

Definition 2.10 A mechanism $\mu=(\sigma, t)$ is a Vickrey-Clarke-Groves (VCG) mechanism if for all $\theta$ and all $i \in N, \sigma(\theta) \in E(\theta)$ and

$$
t_{i}(\theta)=\sum_{j \in N \backslash\{i\}}\left(\sigma_{j}^{0}-\sigma_{j}(\theta)\right) \theta_{j}+g_{i}\left(\theta_{N \backslash\{i\}}\right) .
$$

Writing $\sum_{j \in N \backslash\{i\}}\left(\sigma_{j}^{0}-\sigma_{j}(\theta)\right) \theta_{j}=B(\theta)-\left(\sigma_{i}^{0}-\sigma_{i}(\theta)\right) \theta_{i}$ and $g_{i}\left(\theta_{N \backslash\{i\}}\right)=$ $-B_{-i}^{*}\left(\theta_{N \backslash\{i\}}\right)+h_{i}\left(\theta_{N \backslash\{i\}}\right)$, we can define the VCG mechanisms in the following way.

Definition 2.11 A mechanism $\mu=(\sigma, t)$ is a VCG mechanism if for all $\theta$ and all $i \in N, \sigma(\theta) \in E(\theta)$ and

$$
t_{i}(\theta)=B(\theta)-\left(\sigma_{i}^{0}-\sigma_{i}(\theta)\right) \theta_{i}-B_{-i}^{*}\left(\theta_{N \backslash\{i\}}\right)+h_{i}\left(\theta_{N \backslash\{i\}}\right)
$$

Remark 2.12 The symmetrically balanced VCG mechanism [see Chun et al. (2016), Kayi and Ramaekers (2010) and Mitra (2001)] satisfies BB, OE, SP and equal treatment of equals. ${ }^{10}$ It also satisfies the identical preferences lower bound which requires an agent's utility to be at least her expected utility assuming every queue is chosen with equal probability. When there is no initial queue, this measure provides a "natural" way of defining individual rationality.

Hence, mechanisms satisfying BB, OE, SP and identical preferences lower bound exist when there is no initial queue. One way of interpreting the negative result noted in Remark 2.9 is that it is not possible to design mechanisms satisfying all four axioms when "property rights" in the queue positions are strong. This has also been noted by Gershkov and Schweinzer (2010). ${ }^{11}$

\section{Dropping budget balance}

We start our investigations by characterizing the sub-class of VCG mechanisms satisfying OE, SP and IR. The following lemma, which will be used subsequently, lists the implications of $\mathrm{OE}$.

Lemma 3.1 Let $\mu=(\sigma, t)$ satisfy $O E$. Then, for all $\theta$ and all $i \in N$,

(1) $B(\theta) \geq B_{-i}^{*}\left(\theta_{N \backslash\{i\}}\right)$.

(2) $\sum_{i \in N} B_{-i}^{*}\left(\theta_{N \backslash\{i\}}\right)=(n-2) B(\theta)$.

(3) $B(\theta) \geq B_{i}^{*}\left(\theta_{N \backslash\{i\}}\right) \geq B_{-i}^{*}\left(\theta_{N \backslash\{i\}}\right)$.

\footnotetext{
10 Equal treatment of equals implies that if two agents have the same waiting cost, then they should end up with the same utilities.

11 See also, in a different context, Cramton et al. (1987).
} 
Proof For all $i, j \in N, i \neq j$, let $A_{i j}(\theta)=\left[\sigma_{j}^{0}-\sigma_{j}(\theta)\right]-\left[\sigma_{j}^{0,-i}-\sigma_{j}\left(\theta_{N \backslash\{i\}}\right)\right]=$ $\left[\sigma_{j}^{0}-\sigma_{j}^{0,-i}\right]-\left[\sigma_{j}(\theta)-\sigma_{j}\left(\theta_{N \backslash\{i\}}\right)\right]$. The two terms in $A_{i j}(\theta)$ represents the change to agent $j$ 's queue position when agent $i$ is removed from the queue. The first term $\sigma_{j}^{0}-\sigma_{j}^{0,-i}$ represents the change in agent $j$ 's position in the initial queue and the second term $\sigma_{j}(\theta)-\sigma_{j}\left(\theta_{N \backslash\{i\}}\right)$ represents the change in the reordered queue.

By definition,

$$
B(\theta)-B_{-i}^{*}\left(\theta_{N \backslash\{i\}}\right)=\left(\sigma_{i}^{0}-\sigma_{i}(\theta)\right) \theta_{i}+\sum_{j \in N \backslash\{i\}} A_{i j}(\theta) \theta_{j} .
$$

By OE, $\sum_{j \in N \backslash\{i\}} A_{i j}(\theta)=\left|F_{i}\left(\sigma^{0}\right)\right|-\left|F_{i}(\sigma(\theta))\right|=\left(n-\sigma_{i}^{0}\right)-\left(n-\sigma_{i}(\theta)\right)=$ $-\left(\sigma_{i}^{0}-\sigma_{i}(\theta)\right)$. Hence,

$$
B(\theta)-B_{-i}^{*}\left(\theta_{N \backslash\{i\}}\right)=\sum_{j \in N \backslash\{i\}} A_{i j}(\theta)\left(\theta_{j}-\theta_{i}\right)
$$

We now show that $A_{i j}(\theta)\left(\theta_{j}-\theta_{i}\right)$ is always non-negative.

(i) If $j \in F_{i}\left(\sigma^{0}\right) \cap F_{i}(\sigma(\theta))$ or $j \in P_{i}\left(\sigma^{0}\right) \cap P_{i}(\sigma(\theta))$, then $A_{i j}(\theta)=0$ and so, $A_{i j}(\theta)\left(\theta_{j}-\theta_{i}\right)=0$.

(ii) If $j \in F_{i}\left(\sigma^{0}\right) \cap P_{i}(\sigma(\theta))$, then $A_{i j}(\theta)=1$ and from OE, we get $\theta_{j} \geq \theta_{i}$. Hence, $A_{i j}(\theta)\left(\theta_{j}-\theta_{i}\right) \geq 0$.

(iii) If $j \in P_{i}\left(\sigma^{0}\right) \cap F_{i}(\sigma(\theta))$, then $A_{i j}(\theta)=-1$ and from $\mathrm{OE}$, we get $\theta_{j} \leq \theta_{i}$. Hence, $A_{i j}(\theta)\left(\theta_{j}-\theta_{i}\right) \geq 0$.

This proves (1). For (2), observe that

$$
\begin{aligned}
n B(\theta)-\sum_{i \in N} B_{-i}^{*}\left(\theta_{N \backslash\{i\}}\right) & =\sum_{i \in N}\left(B(\theta)-B_{-i}^{*}\left(\theta_{N \backslash\{i\}}\right)\right) \\
& =\sum_{i \in N}\left(\sigma_{i}^{0}-\sigma_{i}(\theta)\right) \theta_{i}+\sum_{i \in N} \sum_{j \in N \backslash\{i\}} A_{i j}(\theta) \theta_{j} \\
& =B(\theta)+\sum_{i \in N} \sum_{j \in F_{i}\left(\sigma^{0}\right)} \theta_{j}-\sum_{i \in N} \sum_{j \in F_{i}(\sigma(\theta))} \theta_{j} \\
& =B(\theta)+\sum_{i \in N}\left(\sigma_{i}^{0}-1\right) \theta_{i}-\sum_{i \in N}\left(\sigma_{i}(\theta)-1\right) \theta_{i} \\
& =B(\theta)+\sum_{i \in N}\left(\sigma_{i}^{0}-\sigma_{i}(\theta)\right) \theta_{i} \\
& =2 B(\theta) .
\end{aligned}
$$

Finally, for (3), note that a consequence of (1) is that for all $\theta_{N \backslash\{i\}}$ and all $\theta_{i} \in$ $\mathbb{R}_{+}, B\left(\theta_{i}, \theta_{N \backslash\{i\}}\right) \geq B_{-i}^{*}\left(\theta_{N \backslash\{i\}}\right)$. Using the definition of $B_{i}^{*}\left(\theta_{N \backslash\{i\}}\right)$, we conclude that for all $i \in N, B(\theta) \geq B_{i}^{*}\left(\theta_{N \backslash\{i\}}\right) \geq B_{-i}^{*}\left(\theta_{N \backslash\{i\}}\right)$. 
The next result completely characterizes the class of mechanisms satisfying $\mathrm{OE}$, SP and IR.

Proposition 3.2 A mechanism $\mu=(\sigma, t)$ satisfies $O E$, SP and IR if and only if it is a $V C G$ mechanism such that for all $\theta$ and all $i \in N$,

$$
h_{i}\left(\theta_{N \backslash\{i\}}\right) \geq B_{-i}^{*}\left(\theta_{N \backslash\{i\}}\right)-B_{i}^{*}\left(\theta_{N \backslash\{i\}}\right) .
$$

Proof Since $\mu$ satisfies OE and SP, it is a VCG mechanism and has a transfer given by (2.6). Choose $i \in N$ and a profile $\theta=\left(\theta_{i}, \theta_{N \backslash\{i\}}\right)$. The net utility of agent $i$ is

$$
U_{i}\left(\sigma_{i}(\theta), t_{i}(\theta) ; \theta_{i}\right)=B(\theta)-B_{-i}^{*}\left(\theta_{N \backslash\{i\}}\right)+h_{i}\left(\theta_{N \backslash\{i\}}\right) .
$$

For IR, it is both necessary and sufficient that the right-hand side of (3.3) is nonnegative for all $\theta_{i} \in \mathbb{R}_{+}$. By definition, $B_{i}^{*}\left(\theta_{N \backslash\{i\}}\right) \leq B\left(\theta_{i}, \theta_{N \backslash\{i\}}\right)$ for all $\theta_{i} \in \mathbb{R}_{+}$and hence, it is necessary and sufficient that $B_{i}^{*}\left(\theta_{N \backslash\{i\}}\right)-B_{-i}^{*}\left(\theta_{N \backslash\{i\}}\right)+h_{i}\left(\theta_{N \backslash\{i\}}\right) \geq 0$. Or equivalently, $h_{i}\left(\theta_{N \backslash\{i\}}\right) \geq B_{-i}^{*}\left(\theta_{N \backslash\{i\}}\right)-B_{i}^{*}\left(\theta_{N \backslash\{i\}}\right)$. Since the selection of agent $i$ was arbitrary, the result follows.

\subsection{Feasibility and weak feasibility}

The main result of this subsection is to demonstrate that SP, OE and IR are compatible with WF though not with F. Before proving these results, we identify two different VCG mechanisms that have important implications. It can be easily confirmed that both mechanisms satisfy IR as their transfers satisfy (3.2).

Definition 3.3 A mechanism $\mu^{p}=\left(\sigma, t^{p}\right)$ is the pivotal mechanism if for all $\theta$ and all $i \in N, \sigma(\theta) \in E(\theta)$ and

$$
t_{i}^{p}(\theta)=B(\theta)-\left(\sigma_{i}^{0}-\sigma_{i}(\theta)\right) \theta_{i}-B_{-i}^{*}\left(\theta_{N \backslash\{i\}}\right) .
$$

Remark 3.4 The transfers in the pivotal mechanism are obtained from (2.6) by setting $h_{i}\left(\theta_{N \backslash\{i\}}\right)=0$ for all $i \in N$ and all profiles $\theta$. Observe that for all $\sigma^{0}$ and all $\theta$, $U_{i}\left(\sigma_{i}(\theta), t_{i}^{p}(\theta) ; \theta_{i}\right)=B(\theta)-B_{-i}^{*}\left(\theta_{N \backslash\{i\}}\right) \geq 0$ where the equality is by definition of the net utility gain and (3.4) and the inequality is due to Lemma 3.1.

Definition 3.5 A mechanism $\mu^{m d}=\left(\sigma, t^{m d}\right)$ is the minimum deficit VCG mechanism (or MD-VCG mechanism) if for all $\theta$ and all $i \in N, \sigma(\theta) \in E(\theta)$ and

$$
t_{i}^{m d}(\theta)=-\left(\sigma_{i}^{0}-\sigma_{i}(\theta)\right) \theta_{i}+B(\theta)-B_{i}^{*}\left(\theta_{N \backslash\{i\}}\right) .
$$

Remark 3.6 The transfers in the MD-VCG mechanism are obtained from (2.6) by setting $h_{i}\left(\theta_{N \backslash\{i\}}\right)=B_{-i}^{*}\left(\theta_{N \backslash\{i\}}\right)-B_{i}^{*}\left(\theta_{N \backslash\{i\}}\right)$ for all $i \in N$ and all profiles $\theta$. It follows from (2.6) that

$$
\sum_{i \in N} t_{i}(\theta)=(n-1) B(\theta)-\sum_{i \in N} B_{-i}^{*}\left(\theta_{N \backslash\{i\}}\right)+\sum_{i \in N} h_{i}\left(\theta_{N \backslash\{i\}}\right) .
$$


Using Lemma 3.1, this simplifies to

$$
\sum_{i \in N} t_{i}(\theta)=B(\theta)+\sum_{i \in N} h_{i}\left(\theta_{N \backslash\{i\}}\right) .
$$

The budget deficit of a VCG mechanism is thus minimized when the second term on the right-hand side of (3.6) is minimized. It now follows from Proposition 3.2 that the MD-VCG mechanism generates the minimum deficit at all profiles in the sub-class of mechanisms satisfying OE, SP and IR.

Theorem 3.7 OE, SP, IR and $F$ are incompatible.

Proof Let $\left(\sigma, t^{m d}\right)$ be the MD-VCG mechanism. Using Remark 3.6, it suffices to show that there exists a profile $\theta$ such that $\sum_{i \in N} t_{i}^{m d}(\theta)>0$.

Without loss of generality, suppose that $\sigma^{0}$ is such that $\sigma_{i}^{0}=n-i+1$ for all $i \in N$. Let $\theta$ be such that $\theta_{1}>\theta_{n}>\theta_{n-1}>\cdots>\theta_{2}$. The unique efficient queue for this profile is

$$
\sigma_{j}(\theta)= \begin{cases}1 & \text { if } j=1, \\ n-j+2 & \text { if } j \neq 1\end{cases}
$$

Using (3.5), we get $t_{1}^{m d}(\theta)=-\sum_{j=2}^{n} \theta_{j}, t_{n}^{m d}(\theta)=\theta_{1}$, and for $n \geq 3, t_{i}^{m d}(\theta)=$ $\theta_{i+1}$ for all $i \in\{2, \ldots, n-1\}$. Therefore, $\sum_{i \in N} t_{i}^{m d}(\theta)=-\sum_{j=2}^{n} \theta_{j}+\sum_{j=3}^{n} \theta_{j}+$ $\theta_{1}=\theta_{1}-\theta_{2}>0$ and we have the required incompatibility.

Remark 3.8 Theorem 3.7 shows that any mechanism satisfying OE, SP and IR runs a budget deficit at some profile. Furthermore, the extent of the budget deficit is unbounded both in absolute and in per capita terms. Note that in the proof of Theorem 3.7, the budget deficit is the difference between $\theta_{1}$ and $\theta_{2}$ and this can be made as large as one wants.

Obtaining a possibility result requires weakening the notion of feasibility further to weak feasibility. The motivation for weak feasibility is clear. The budget deficit is being incurred to achieve a saving in waiting costs. Hence, we should look at the difference between the two and not the budget deficit in isolation. ${ }^{12}$

Theorem 3.9 A mechanism $\mu=(\sigma, t)$ satisfies $O E, S P, I R$ and WF if and only if it is a VCG mechanism such that for all $\theta$ and all $i \in N, h_{i}\left(\theta_{N \backslash\{i\}}\right) \geq B_{-i}^{*}\left(\theta_{N \backslash\{i\}}\right)-$ $B_{i}^{*}\left(\theta_{N \backslash\{i\}}\right)$ and $\sum_{i \in N} h_{i}\left(\theta_{N \backslash\{i\}}\right) \leq 0$.

Proof Follows from Proposition 3.2 and (3.6).

Remark 3.10 For the pivotal mechanism, $\sum_{i \in N} h_{i}\left(\theta_{N \backslash\{i\}}\right)=0$ for all $\theta$. This represents one extreme corresponding to the maximum subsidy given to the agents. From (3.6), it is clear that the subsidy equals the cost saving from moving to an efficient queue. The other extreme, corresponding to the minimum subsidy, is represented by the MD-VCG mechanism. All OE, SP, IR and WF mechanisms will have (for each profile) subsidies in between these extremes.

12 We are grateful to Larry Samuelson for making this point to us. 


\section{Dropping outcome efficiency}

We start by looking at the $n=2$ case and then proceeding to the $n \geq 3$ case.

\subsection{The two-agent case}

There are two possible initial queues here, one with agent 1 is in the first position (call this $\sigma^{0}$ ) and one with agent 2 is in the first position (call this $\sigma^{1}$ ). We assume, without loss of generality, that $\sigma^{0}$ is the initial queue. We show that the only mechanisms which satisfy BB, SP and IR are constant or fixed-price. The former selects the initial queue and assigns zero transfers at all profiles. In the latter, a price $p$ is chosen a priori. The two agents exchange positions if they both desire it, in which case the agent moving forward pays $p$ to the agent moving back.

Definition 4.1 Let $N=\{1,2\}$ and $\sigma^{0}$ be the initial queue. A mechanism $\mu=(\sigma, t)$ is constant if for all $\theta$ and all $i \in N, \sigma(\theta)=\sigma^{0}$ and $t_{1}(\theta)=t_{2}(\theta)=0$.

Definition 4.2 Let $N=\{1,2\}$ and $\sigma^{0}$ be the initial queue. The mechanism $\mu=(\sigma, t)$ is fixed-price if there exists $p \geq 0$ such that the following conditions hold.

FP1. If $\theta_{1}<p<\theta_{2}, \sigma(\theta)=\sigma^{1}$.

FP2. If $\theta_{1}>p$ or $\theta_{2}<p$, then $\sigma(\theta)=\sigma^{0}$.

FP3. (a) Either (i) $\sigma\left(p, \theta_{2}\right)=\sigma^{0}$ for all $\theta_{2} \geq p$ or (ii) $\sigma\left(p, \theta_{2}\right)=\sigma^{1}$ for all $\theta_{2}>p$ and $\sigma(p, p)$ is either $\sigma^{0}$ or $\sigma^{1}$.

(b) Either (i) $\sigma\left(\theta_{1}, p\right)=\sigma^{0}$ for all $\theta_{1} \leq p$ or (ii) $\sigma\left(\theta_{1}, p\right)=\sigma^{1}$ for all $\theta_{1}<p$ and $\sigma(p, p)$ is either $\sigma^{0}$ or $\sigma^{1}$.

FP4. For all $\theta$ such that $\sigma(\theta)=\sigma^{1}, t_{1}(\theta)=-t_{2}(\theta)=p$, and for all $\theta$ such that $\sigma(\theta)=\sigma^{0}, t_{1}(\theta)=t_{2}(\theta)=0$.

Remark 4.3 FP4 says that when the queue is reordered, the agent moving forward pays $p$ to the one moving back; otherwise no transfers are given or paid. The other three rules specify the conditions under which the queue is reordered. FP1 says that a reordering takes place if both agents strictly benefit from it. FP2 says that no reordering takes place if at least one agent strictly loses from the reordering. FP3(a) looks at profiles where agent 1 is indifferent between the initial queue and the reordered queue. This happens when $\theta_{1}=p$. In this case, there are two possibilities: either no reordering takes place for all $\theta_{2} \geq p$ or reordering takes place for all $\theta_{2}>p$ and either queue is possible when $\theta_{2}=p$. FP3(b) is the symmetric case when agent 2 is indifferent between the initial queue and the reordered queue.

Lemma 4.4 Let $N=\{1,2\}$ and $\sigma^{0}$ be the initial queue. Let $\mu=(\sigma, t)$ satisfy $B B$, $S P$ and IR. Let $\theta$ be a profile and denote $p(\theta)=t_{1}(\theta)$. Then

(1) $p(\theta)=t_{1}(\theta)=-t_{2}(\theta)$.

(2) $p(\theta) \geq 0$ and $p(\theta)=0$ if $\sigma(\theta)=\sigma^{0}$.

Proof The first part follows from BB. For the second part, first suppose that $\sigma(\theta)=\sigma^{1}$. Then, IR for agent 1 implies that $p(\theta)-\theta_{1} \geq 0$ or $p(\theta) \geq \theta_{1} \geq 0$. If $\sigma(\theta)=\sigma^{0}$, 
then IR for agents 1 and 2 both imply that $t_{1}(\theta)=t_{2}(\theta) \geq 0$. By BB, it follows that $t_{1}(\theta)=t_{2}(\theta)=p(\theta)=0$.

Theorem 4.5 Let $N=\{1,2\}$ and $\sigma^{0}$ be the initial queue. A mechanism $\mu=(\sigma, t)$ satisfies BB, SP and IR if and only if it is either a constant or a fixed-price mechanism.

Proof We only prove necessity since sufficiency is straightforward. Suppose $\mu=$ $(\sigma, t)$ satisfies BB, SP and IR. There are two possibilities. First, if $\sigma(\theta)=\sigma^{0}$ for all $\theta$, then Lemma 4.4 implies that $p(\theta)=0$ for all $\theta$ and thus $\mu$ is a constant mechanism.

The second possibility is that there exists $\theta^{\prime}$ such that $\sigma\left(\theta^{\prime}\right)=\sigma^{1}$. Denote $p\left(\theta^{\prime}\right)=$ $p$. We now work out the allocations at all profiles $\theta \neq \theta^{\prime}$. There are eight possible cases. Assume first that $p>0$.

Case 1: $\theta_{1}>\theta_{2}$

Suppose $\sigma(\theta)=\sigma^{1}$. IR for agent 1 implies that $p(\theta)-\theta_{1} \geq 0$ or $p(\theta) \geq \theta_{1}$. IR for agent 2 implies that $-p(\theta) \geq-\theta_{2}$ or $p(\theta) \leq \theta_{2}$. Hence, $\theta_{1} \leq p(\theta) \leq \theta_{2}$ which contradicts $\theta_{1}>\theta_{2}$. Thus, $\sigma(\theta)=\sigma^{0}$ and by Lemma 4.4, $p(\theta)=0$.

Case 2: $\theta_{1} \in[0, p), \theta_{2}=\theta_{2}^{\prime}$.

If $\sigma(\theta)=\sigma^{0}$, then by Lemma 4.4, $p(\theta)=0$ and hence, $u_{1}\left(\sigma_{1}(\theta), t_{1}(\theta) ; \theta_{1}\right)=0$. However, agent 1 can deviate unilaterally from $\theta$ to $\theta^{\prime}$ and this deviation is profitable because $u_{1}\left(\sigma_{1}\left(\theta^{\prime}\right), t_{1}\left(\theta^{\prime}\right) ; \theta_{1}\right)=p-\theta_{1}>0$. This violates SP and so, $\sigma(\theta)=\sigma\left(\theta^{\prime}\right)=$ $\sigma^{1}$. If $p(\theta) \neq p$, then agent 1 will manipulate at either $\theta$ or $\theta^{\prime}$ and so, $p(\theta)=p$.

Case 3: $\theta_{2}>p>\theta_{1}$.

If $\sigma(\theta)=\sigma^{0}$, then $p(\theta)=0$ by Lemma 4.4 and so, $u_{2}\left(\sigma_{2}(\theta), t_{2}(\theta) ; \theta_{2}\right)=0$. Let agent 2 deviate from $\theta$ to $\theta^{\prime \prime}=\left(\theta_{1}, \theta_{2}^{\prime}\right)$. By Case $2, \sigma\left(\theta^{\prime \prime}\right)=\sigma^{1}$ and $p\left(\theta^{\prime \prime}\right)=p$. Hence, $u_{2}\left(\sigma\left(\theta^{\prime}\right), t_{2}\left(\theta^{\prime}\right) ; \theta_{2}\right)=-p>-\theta_{2}$. This violates SP and shows that $\sigma(\theta)=\sigma^{1}$. It follows that $p(\theta)=p$; otherwise, agent 2 will manipulate at either $\theta$ or $\theta^{\prime \prime}$.

Case 4: $\theta_{2}>\theta_{1}>p$.

Suppose $\sigma(\theta)=\sigma^{1}$. By BB and IR, $\theta_{1} \leq p(\theta) \leq \theta_{2}$. Hence, $p(\theta) \geq \theta_{1}>p$. This violates SP because agent 1 can deviate profitably from a profile of Case 3 to $\theta$. Hence, $\sigma(\theta)=\sigma^{0}$ and by Lemma 4.4, $p(\theta)=0$.

Case 5: $p>\theta_{2}>\theta_{1}$.

Suppose $\sigma(\theta)=\sigma^{1}$. By BB and IR, $\theta_{1} \leq p(\theta) \leq \theta_{2}$ and so, $p(\theta)<p$. This violates SP because agent 2 can now deviate profitably from a profile of Case 3 to the profile $\theta$. Again, $\sigma(\theta)=\sigma^{0}$ and $p(\theta)=0$.

Case 6: $\theta_{1}=\theta_{2} \neq p$.

If $\sigma(\theta)=\sigma^{1}$, then by BB and IR, $p(\theta)=\theta_{1}=\theta_{2}$. If $p(\theta)>p$ then agent 1 can unilaterally deviate from a profile of Case 3 to $\theta$. If $p(\theta)<p$, then agent 2 can profitably deviate from a profile of Case 3 to $\theta$. Hence, $\sigma(\theta)=\sigma^{0}$ and $p(\theta)=0$.

Case 7: $\theta_{2} \geq \theta_{1}=p$.

Agent 1 cannot profitably deviate from such a profile. Indeed, a deviation will lead to one of Cases 1, 2, 3, 4 or $6 .{ }^{13}$ In Cases 2 and 3, there is trade but at a price of $p$. In the other cases, there is no trade. In all cases, the net utility after deviation is zero and this shows that the agent does not benefit strictly.

Let $\theta$ be a profile such that $\theta_{1}=p$ and $\theta_{2}>p$. Suppose that $\sigma(\theta)=\sigma^{1}$. By BB and IR, $p \leq p(\theta) \leq \theta_{2}$. If $p(\theta)>p$, then agent 1 can profitably deviate at $\theta$ by

13 See Fig. 1. 
announcing $\theta_{1}^{\prime}<p$ (see Case 3). Hence, $p(\theta)=p$. We claim now that $\sigma\left(\theta^{\prime}\right)=\sigma^{1}$ for all profiles $\theta^{\prime}$ such that $\theta_{1}^{\prime}=p$ and $\theta_{2}^{\prime}>p$. Indeed, if $\sigma\left(\theta^{\prime}\right)=\sigma^{0}$, then agent 2 can profitably deviate from $\theta^{\prime}$ to $\theta$.

We have thus shown that if the queue is reordered for some $\theta_{2}>p$, then it has to be reordered for all such $\theta_{2}$. Furthermore, the transaction takes place at a price of $p$. Hence, there are three possibilities which are enumerated below.

(1) For all profiles $\theta$ such that $\theta_{2} \geq \theta_{1}=p, \sigma(\theta)=\sigma^{0}, t_{1}(\theta)=-t_{2}(\theta)=0$.

(2) For all profiles $\theta$ such that $\theta_{2} \geq \theta_{1}=p, \sigma(\theta)=\sigma^{1}, t_{1}(\theta)=-t_{2}(\theta)=p$.

(3) For all profiles $\theta$ such that $\theta_{2}>\theta_{1}=p, \sigma(\theta)=\sigma^{1}, t_{1}(\theta)=-t_{2}(\theta)=p$ but $\sigma(p, p)=\sigma^{0}, t_{1}(p, p)=-t_{2}(p, p)=0$.

Case 8: $\theta_{2}=p \geq \theta_{1}$

The analysis of this case parallels that of Case 7 and is omitted. The equivalent possibilities here are as follows.

(1) For all profiles such that $p=\theta_{2} \geq \theta_{1}, \sigma(\theta)=\sigma^{0}, t_{1}(\theta)=-t_{2}(\theta)=0$.

(2) For all profiles such that $p=\theta_{2} \geq \theta_{1}, \sigma(\theta)=\sigma^{1}, t_{1}(\theta)=-t_{2}(\theta)=p$.

(3) For all profiles such that $p=\theta_{2}>\theta_{1}, \sigma(\theta)=\sigma^{1}, t_{1}(\theta)=-t_{2}(\theta)=p$ but $\sigma(p, p)=\sigma^{0}, t_{1}(p, p)=-t_{2}(p, p)=0$.

Collating the various cases, it is straightforward to verify that the resulting allocations correspond to a fixed-price trading mechanism at the price $p>0$.

Finally, consider the case where $p=0$. In this case, trade can take place only when $\theta_{1}=0$, corresponding to Case 7 . No trade takes place otherwise. This completes the proof.

The operation of the fixed-price mechanism is illustrated in Fig. 1. Below the $45^{\circ}$ line, $\theta_{1}>\theta_{2}$; hence, the initial queue $\sigma^{0}$ is efficient and no trade (of queue positions) takes place. Above this line, trade is efficient but this does not occur for all profiles. Efficient trade requires $\theta_{2} \geq p \geq \theta_{1}$ additionally and this leads to inefficiency for any choice of $p$.

Remark 4.6 Hagerty and Rogerson (1987) prove a similar result to Theorem 4.5 in a related mechanism design problem involving the sale of a unit of a homogeneous good with a single buyer and seller. They show that posted-price mechanisms are essentially the only mechanisms satisfying BB, SP and IR. ${ }^{14}$ Our work differs from their work in two ways. First, the IR constraint is type dependent for both agents in our problem while it is type independent for the buyer in Hagerty and Rogerson (1987). Second, they allow for probabilistic mechanisms while we consider only deterministic mechanisms. Hence, our proof procedure is significantly different.

\subsection{The three or more agent case}

In general, fixed-price mechanisms are characterized by a vector of prices $\left(p_{1}, \ldots, p_{n}\right)$ where $p_{i}$ is the "price" of the $i$ th queue position. For each profile $\theta$, the mechanism

\footnotetext{
14 A posted-price mechanism is one where a price is posted in advance and trade occurs if and only if all traders agree to trade.
} 
Fig. 1 The $n=2$ case when $\sigma_{i}^{0}=i, i=1,2$

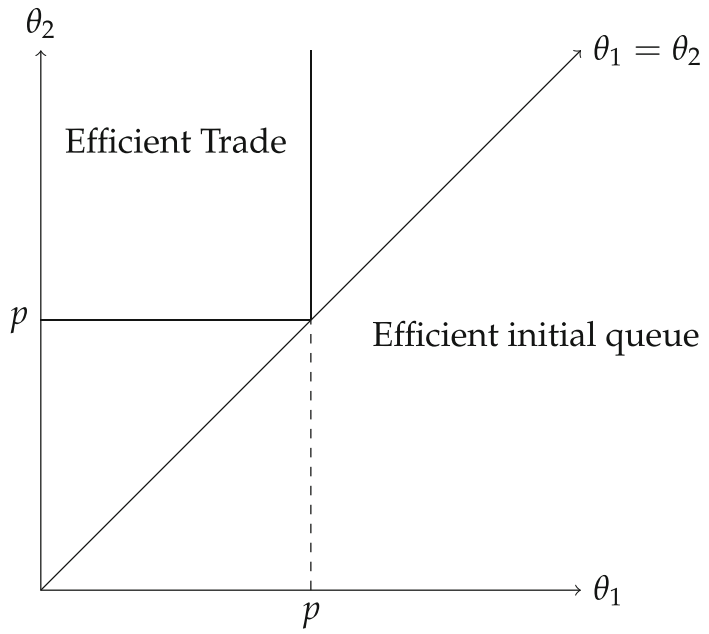

chooses a queue $\sigma(\theta)$ and the transfers are given by $t_{i}(\theta)=p_{\sigma_{i}^{0}}-p_{\sigma_{i}(\theta)}$ for all $i \in N$. Hence, each agent receives as transfer the difference between the price of her final queue position and the price of her initial queue position. The mechanism is budget balanced by definition.

Since the transfers are automatically determined once the queue is chosen, the problem reduces to one of choosing the queue in a manner which preserves SP and IR. To see how this can be done, note that the prices $\left(p_{1}, \ldots, p_{n}\right)$ determine an agent's ordinal preference over different queue positions. In particular, for $\sigma_{i} \in\{1, \ldots, n\}$, define $u_{i}\left(\sigma_{i}\right)=-\left(\sigma_{i}-1\right) \theta_{i}+p_{\sigma_{i}^{0}}-p_{\sigma_{i}}$ to be the utility obtained by agent $i$ if she gets queue position $\sigma_{i}$. The agent's ordinal preference over queue positions is determined by the vector $\left(u_{i}(1), \ldots, u_{i}(n)\right)$. The problem of determining the queue from the ordinal preferences of agents over queue positions is identical to the house allocation problem for which different mechanisms satisfying SP and IR are known to exist. We can choose any of them like the top trading cycle [see Gale and Shapley (1962), Roth (1982) and Shapley and Scarf (1974) among others].

Unlike the two-agent case, constant and fixed-price mechanisms do not exhaust the set of BB, SP and IR mechanisms. We are unable to completely characterize the set of mechanisms satisfying BB, SP and IR. As noted in the introduction, the problem is due to the specific domain restriction that is involved in this problem. What we do is to identify one mechanism which is neither constant nor fixed-price called the median waiting cost exchange mechanism. We will describe this mechanism shortly but we turn now to the question of efficiency.

It follows from Theorem 3.7 that mechanisms satisfying SP, BB and IR are all inefficient meaning that for a given mechanism, there is some profile where the maximum possible cost saving from reordering may not be realized. Hence, the extent of inefficiency of any such mechanism is an important issue. Since our objective is to minimize total waiting cost, a natural measure of efficiency (for a given profile) is 
the fraction of maximum possible waiting cost saving achieved by the mechanism. ${ }^{15}$ However, computing this ratio for a profile is not straightforward since it depends on the initial queue. ${ }^{16}$

We therefore opt for a different approach and focus on the inefficiency of the queue. To understand our measure, note that if the queue is not efficient for a profile $\theta$, then for at least one agent, the queue position allotted by the mechanism $\sigma_{i}(\theta)$ and the agent's position in the efficient queue $\sigma_{i}^{e}(\theta)$ must be different.

Definition 4.7 Let $k \in\{0,1, \ldots, n-1\}$. The mechanism $\mu=(\sigma, t)$ is $k$-queue inefficient if

(1) for all $\theta$ such that $E(\theta)=\left\{\sigma^{e}(\theta)\right\}$ and all $i \in N,\left|\sigma_{i}(\theta)-\sigma_{i}^{e}(\theta)\right| \leq k$, and

(2) there exists $\theta$ such that $E(\theta)=\left\{\sigma^{e}(\theta)\right\}$ and $i \in N$ such that $\left|\sigma_{i}(\theta)-\sigma_{i}^{e}(\theta)\right|=k$.

Remark 4.8 The first condition in the above definition requires that at all profiles where the efficient queue is unique, every agent is within a distance $k$ from her position in the efficient queue. The second condition says that there is at least one profile such that some agent's distance from her position in the efficient queue is equal to $k$.

Remark 4.9 Note that our measure of queue inefficiency only looks at profiles where the efficient queue is unique. This is because the measure depends on the tie-breaking rule when the efficient queue is not unique. To illustrate, consider the profile $\theta$ such that $\theta_{1}>\theta_{2}=\theta_{3}$. Denote the two efficient queues at this profile as $\sigma^{e}$ and $\sigma^{f}$ where $\left(\sigma_{1}^{e}, \sigma_{2}^{e}, \sigma_{3}^{e}\right)=(1,2,3),\left(\sigma_{1}^{f}, \sigma_{2}^{f}, \sigma_{3}^{f}\right)=(1,3,2)$. Suppose the mechanism $\mu$ selects the queue $\sigma(\theta)=\left(\sigma_{1}(\theta), \sigma_{2}(\theta), \sigma_{3}(\theta)\right)=(2,1,3)$. Observe that if $\sigma^{e}$ is selected as the efficient queue by the tie-breaking rule, then $\left|\sigma_{i}(\theta)-\sigma_{i}^{e}(\theta)\right| \leq 1$ for all $i \in N$. However, if $\sigma^{f}$ is selected, then $\left|\sigma_{2}(\theta)-\sigma_{2}^{f}(\theta)\right|=2$.

There are different possibilities for dealing with such profiles but restricting the measure to profiles where the efficient queue is unique is simpler, in our view. This is because the set of profiles where the efficient queue is not unique-that is, $\{\theta \in$ $\mathbb{R}_{+}^{n} \mid \exists i, j \in N, i \neq j$ such that $\left.\theta_{i}=\theta_{j}\right\}$-is a set of measure zero. ${ }^{17}$ Thus, our measure applies to "almost all" profiles.

Remark 4.10 Since the maximal distance between an agent's position in the efficient queue and her actual position is at most $n-1$, every mechanism is at most $(n-1)$-queue inefficient.

Definition 4.11 A mechanism $\mu$ is totally queue inefficient if it is $(n-1)$-queue inefficient.

It is not difficult to see that constant mechanisms are totally queue inefficient. So are fixed-price mechanisms. Indeed, suppose that agent 1 occupies the first position in

\footnotetext{
15 See Eq. (2.1).

16 For the median waiting cost exchange mechanism, it also depends on the order in which agents are allowed to trade: see Definition 4.15.

17 Given $i, j, i \neq j$, the hyperplane $\left\{\theta \in \mathbb{R}_{+}^{n} \mid \theta_{i}=\theta_{j}\right\}$ is a set of measure zero. The finite union of all sets of this type is thus also a set of measure zero.
} 
the initial queue. Let $\left(p_{1}, \ldots, p_{n}\right)$ be the price vector. Consider the profile $0<\theta_{1}<$ $\cdots<\theta_{n}$ where

$$
\theta_{1}>\max \left\{\frac{p_{1}-p_{n}}{n-1}, \ldots, \frac{p_{1}-p_{2}}{2-1}\right\} .
$$

Then, agent 1 will refuse to exchange her position because $-(k-1) \theta_{i}+p_{1}-p_{k}<0$ for all $k \neq 1$. Any queue resulting after trade among other agents will always have agent 1 at a distance of $n-1$ from her position in the unique efficient queue. In contrast, we show that the median waiting cost mechanism is $(n-1) / 2$-queue inefficient if $n$ is odd and $n / 2$-queue inefficient if $n$ is even.

Definition 4.12 Let $n \geq 3$ and odd. ${ }^{18}$ Given a profile $\theta$, an agent $i_{m} \in N$ is a median agent if

$$
\left|\left\{i: \theta_{i} \geq \theta_{i_{m}}\right\}\right| \geq \frac{n+1}{2} \text { and }\left|\left\{i: \theta_{i} \leq \theta_{i_{m}}\right\}\right| \geq \frac{n+1}{2}
$$

The waiting cost of a median agent, denoted $\theta_{m}$, is the median waiting cost. ${ }^{19}$

Let $M(\theta) \equiv\left\{i \in N \mid \theta_{i}>\theta_{m}\right\}$ be the set of agents with waiting costs strictly greater than $\theta_{m}$. Similarly, let $m(\theta) \equiv\left\{i \in N \mid \theta_{i}<\theta_{m}\right\}$ be the set of agents with waiting costs strictly smaller than $\theta_{m}$.

Definition 4.13 Let $\theta$ be a profile and $\sigma$ a queue. Let $\{i, j\}$ be a pair of distinct agents. An exchange is feasible between $i$ and $j$ if

(1) $\theta_{j}>\theta_{m}>\theta_{i}$ and $\sigma_{i}<\sigma_{j}$, or

(2) $\theta_{j}=\theta_{m}>\theta_{i}, \sigma_{i}<\sigma_{j}$ and $M(\theta)=\emptyset$, or

(3) $\theta_{j}>\theta_{m}=\theta_{i}, \sigma_{i}<\sigma_{j}$ and $m(\theta)=\emptyset$.

Remark 4.14 Given a queue $\sigma$, an exchange of queue positions between two agents, $i$ and $j$, will reduce waiting cost if $\sigma_{i}>\sigma_{j}$ and $\theta_{i}>\theta_{j} .{ }^{20}$ A feasible exchange requires additionally that $\theta_{i}>\theta_{m}>\theta_{j}$. Hence, some exchanges which decrease waiting cost are not feasible. We need feasibility to maintain strategyproofness.

The second and third conditions in Definition 4.13 relate to agents whose waiting cost equals the median waiting cost. Such agents are not part of a feasible exchange unless the median waiting cost is also the highest (or the lowest) waiting cost.

Let $\succ$ be a linear order on the set of unordered pairs of the set $N .^{21}$ Let $n^{*}=$ $n(n-1) / 2$.

Definition 4.15 Let $n \geq 3$ and odd. Let $\sigma^{0}$ be the initial queue and $t_{i}^{0}=0$ for all $i \in N$. The median waiting cost exchange mechanism $\mu^{m}$ is defined by the following algorithm.

\footnotetext{
18 We discuss how this mechanism can be modified to handle a problem with an even number of agents in Sect. 4.3.

19 While there may be more than one median agent, all such agents have the same waiting cost.

20 The amount of waiting cost saving is $\left(\sigma_{i}-\sigma_{j}\right)\left(\theta_{i}-\theta_{j}\right)$.

21 As is conventional, we assume that if $\{a, b\}$ is an unordered pair, then $a \neq b$.
} 
At stage $k \geq 1$, the input to the algorithm is the profile $\theta$, the queue $\sigma^{k-1}$, the previous cumulative transfer vector $t^{k-1}$ and the $k$ th element of the order $\succ$, say, $\left\{i_{k}, j_{k}\right\}$.

(1) If there is no feasible exchange between $i_{k}$ and $j_{k}$, then $\sigma^{k}=\sigma^{k-1}$ and $t^{k}=t^{k-1}$.

(2) Otherwise, define the queue $\sigma^{k}$ and the transfers $t^{k}$ by

$$
\left(\sigma_{i}^{k}, t_{i}^{k}\right)= \begin{cases}\left(\sigma_{i}^{k-1}, t_{i}^{k-1}\right) & \text { if } i \neq i_{k}, j_{k}, \\ \left(\sigma_{i_{k}}^{k-1}, t_{i}^{k-1}+\left(\sigma_{i_{k}}^{k-1}-\sigma_{j_{k}}^{k-1}\right) \theta_{m}\right) & \text { if } i=j_{k}, \\ \left(\sigma_{j_{k}}^{k-1}, t_{i}^{k-1}+\left(\sigma_{j_{k}}^{k-1}-\sigma_{i_{k}}^{k-1}\right) \theta_{m}\right) & \text { if } i=i_{k} .\end{cases}
$$

Remark 4.16 The algorithm converges since there are a finite number of agents. At stage $k$, agents $i_{k}$ and $j_{k}$ are given an opportunity to exchange their queue positions. They do so if the exchange benefits both of them. If there is an exchange, then agent $i_{k}$ pays $\left(\sigma_{j_{k}}^{k-1}-\sigma_{i_{k}}^{k-1}\right) \theta_{m}$ to agent $j_{k}$. Otherwise, the queue remains unchanged and no transfers take place.

Remark 4.17 Observe that agents with waiting costs strictly higher (lower) than the median waiting cost can move forward (backward) in the queue, or remain stationary at each step. Hence, if $\theta_{i} \gtrless \theta_{m}$ then $\sigma_{i}(\theta) \lesseqgtr \sigma_{i}^{0}$.

What happens if $\theta_{i}=\theta_{m}$ ? There are three possibilities. First, suppose that $M(\theta) \neq$ $\emptyset$ and $m(\theta) \neq \emptyset$. In this case, agent $i$ is never a part of any feasible exchange and hence, $\sigma_{i}(\theta)=\sigma_{i}^{0}$. Second, we might have $M(\theta)=\emptyset, m(\theta) \neq \emptyset$. That is, agent $i$ is also an agent with the highest waiting cost. Here, Rule 2 of Definition 4.13 applies and a feasible exchange can involve agent $i$. Such exchanges can only move agent $i$ forward in the queue, and so, $\sigma_{i}(\theta) \leq \sigma_{i}^{0}$. If $M(\theta) \neq \emptyset$ and $m(\theta)=\emptyset$, then Rule 3 applies; agent $i$ can only move backwards at each step (involving her) and we have $\sigma_{i}(\theta) \geq \sigma_{i}^{0} .22$

Remark 4.18 The mechanism $\mu^{m}$ satisfies BB by construction and IR because a feasible exchange takes place only if it (weakly) benefits both agents. Since all pairwise exchanges occur at the "price" of $\theta_{m}$, the ex post utility of agent $i$ is

$$
u_{i}\left(\sigma_{i}(\theta), t_{i}(\theta) ; \theta_{i}\right)=-\left(\sigma_{i}(\theta)-1\right) \theta_{i}+\left(\sigma_{i}(\theta)-\sigma_{i}^{0}\right) \theta_{m}
$$

Theorem 4.19 Let $n>2$ and odd. Then, the median waiting cost exchange mechanism $\mu^{m}$ satisfies $B B, S P, I R$ and is $(n-1) / 2$-queue inefficient.

Proof BB and IR follow from Remark 4.18. For SP, let $\theta$ and $\theta^{\prime}$ be $i$-variants. Using (4.1), we can compute the benefit to agent $i$ of a deviation from $\theta$ to $\theta^{\prime}$ to be

$$
\Delta=\left(\sigma_{i}(\theta)-\sigma_{i}\left(\theta^{\prime}\right)\right) \theta_{i}-\left(\sigma_{i}(\theta)-\sigma_{i}^{0}\right) \theta_{m}+\left(\sigma_{i}\left(\theta^{\prime}\right)-\sigma_{i}^{0}\right) \theta_{m}^{\prime}
$$

\footnotetext{
22 In addition to the three cases, there is additionally a trivial case where $M(\theta)=m(\theta)=\emptyset$. This corresponds to the case where all agents have the same waiting cost. In this case, all queues are efficient.
} 
Note that in (4.2), $\theta_{m}$ and $\theta_{m}^{\prime}$ are the median waiting costs for the profiles $\theta$ and $\theta^{\prime}$. We will show that $\Delta \leq 0$ in all cases.

Case $1 \min \left\{\theta_{i}, \theta_{i}^{\prime}\right\}>\theta_{m}$ or $\max \left\{\theta_{i}, \theta_{i}^{\prime}\right\}<\theta_{m}$.

In both cases, $\theta_{m}=\theta_{m}^{\prime}$. It follows from Definition 4.15 that $\sigma_{i}(\theta)=\sigma_{i}\left(\theta^{\prime}\right)$ and $t_{i}(\theta)=t_{i}\left(\theta^{\prime}\right)$. Hence, $\Delta=0$.

Case $2 \theta_{i}^{\prime}>\theta_{m}>\theta_{i}$.

Observe that $\theta_{i}^{\prime} \geq \theta_{m}^{\prime} \geq \theta_{m}$. By Remark 4.17, $\sigma_{i}(\theta) \geq \sigma_{i}^{0}$. If $\theta_{i}^{\prime}>\theta_{m}^{\prime}$, then by Remark 4.17 again, $\sigma_{i}\left(\theta^{\prime}\right) \leq \sigma_{i}^{0}$. Using (4.2), we have

$$
\begin{aligned}
\Delta & =\left(\sigma_{i}(\theta)-\sigma_{i}\left(\theta^{\prime}\right)\right) \theta_{i}-\left(\sigma_{i}(\theta)-\sigma_{i}^{0}\right) \theta_{m}+\left(\sigma_{i}\left(\theta^{\prime}\right)-\sigma_{i}^{0}\right) \theta_{m}^{\prime} \\
& \leq\left(\sigma_{i}(\theta)-\sigma_{i}\left(\theta^{\prime}\right)\right) \theta_{i}-\left(\sigma_{i}(\theta)-\sigma_{i}^{0}\right) \theta_{m}+\left(\sigma_{i}\left(\theta^{\prime}\right)-\sigma_{i}^{0}\right) \theta_{m} \\
& =\left(\sigma_{i}(\theta)-\sigma_{i}\left(\theta^{\prime}\right)\right)\left(\theta_{i}-\theta_{m}\right) \\
& \leq 0 .
\end{aligned}
$$

To complete the argument for this case, suppose that $\theta_{i}^{\prime}=\theta_{m}^{\prime}$. If $\sigma_{i}\left(\theta^{\prime}\right)>\sigma_{i}^{0}$, then $m\left(\theta^{\prime}\right)=\emptyset$ by Remark 4.17. Since $\theta_{m}^{\prime} \geq \theta_{m}$, this implies $\theta_{i}^{\prime}=\theta_{m}^{\prime}=$ $\theta_{m}<\theta_{i}^{\prime}$, a contradiction. Hence, $\sigma_{i}\left(\theta^{\prime}\right) \leq \sigma_{i}^{0}$, but then (4.3) again follows.

Case $3 \theta_{i}>\theta_{m}>\theta_{i}^{\prime}$.

The analysis of this case is similar to Case 2 and is omitted.

Case $4 \theta_{i}>\theta_{i}^{\prime}=\theta_{m}$.

Observe that $\theta_{m}^{\prime}=\theta_{m}, m\left(\theta^{\prime}\right)=m(\theta)$. Furthermore, $M(\theta) \neq \varnothing$ since $i \in$ $M(\theta)$. From (4.2), we get $\Delta=\left(\sigma_{i}\left(\theta^{\prime}\right)-\sigma_{i}(\theta)\right)\left(\theta_{m}-\theta_{i}\right)$. Hence, the deviation is profitable only if $\sigma_{i}\left(\theta^{\prime}\right)<\sigma_{i}(\theta)$. We will show that this is not the case.

(a) If $m(\theta)=m\left(\theta^{\prime}\right)=\emptyset$, then $\sigma_{i}\left(\theta^{\prime}\right) \geq \sigma_{i}^{0}$ and $\sigma_{i}(\theta) \leq \sigma_{i}^{0}$ by Remark 4.17. Hence, $\sigma_{i}\left(\theta^{\prime}\right) \geq \sigma_{i}(\theta)$.

(b) If $m(\theta)=m\left(\theta^{\prime}\right) \neq \emptyset$ and $M\left(\theta^{\prime}\right) \neq \emptyset$, then it follows from Remark 4.17 that (i) $\sigma_{i}\left(\theta^{\prime}\right)=\sigma_{i}^{0}$ and (ii) $\sigma_{i}(\theta) \leq \sigma_{i}^{0}$. Again, $\sigma_{i}\left(\theta^{\prime}\right) \geq \sigma_{i}(\theta)$

(c) Finally, suppose that $m\left(\theta^{\prime}\right)=m(\theta) \neq \emptyset$ and $M\left(\theta^{\prime}\right)=\emptyset$. Suppose there is a feasible exchange between $i$ and $j$ at stage $k$ in the profile $\theta^{\prime}$. Since $M\left(\theta^{\prime}\right)=\emptyset$, it follows from Definition 4.13 that $j \in m\left(\theta^{\prime}\right)$ and $\sigma_{0}^{j}<\sigma_{0}^{i}$. Since $i \in M(\theta)$ and $m(\theta)=m\left(\theta^{\prime}\right)$, it follows that the same exchange is feasible at stage $k$ in the profile $\theta$. Hence, $\sigma_{i}(\theta) \leq \sigma_{i}\left(\theta^{\prime}\right){ }^{23}$

Case $5 \theta_{i}<\theta_{i}^{\prime}=\theta_{m}$.

The analysis of this case closely follows from Case 4 and is omitted.

Case $6 \theta_{i}=\theta_{m}>\theta_{i}^{\prime}$.

Note that $\theta_{i}^{\prime} \leq \theta_{m}^{\prime} \leq \theta_{m}=\theta_{i}$. Using (4.2), we have $\Delta=\left(\sigma_{i}\left(\theta^{\prime}\right)-\sigma_{i}^{0}\right)\left(\theta_{m}^{\prime}-\right.$ $\left.\theta_{m}\right)$ and so, the deviation is beneficial only if $\sigma_{i}\left(\theta^{\prime}\right)<\sigma_{0}^{i}$. If $\theta_{i}^{\prime}<\theta_{m}^{\prime}$ then

\footnotetext{
23 There is an inequality because there might be additional feasible exchanges involving $i$ in the profile $\theta$. By Remark 4.17, such exchanges must move agent $i$ ahead in the queue.
} 
$\sigma_{i}\left(\theta^{\prime}\right) \geq \sigma_{i}^{0}$ by Remark 4.17. On the other hand, if $\theta_{i}^{\prime}=\theta_{m}^{\prime}$, then $M\left(\theta^{\prime}\right) \neq \emptyset .{ }^{24}$ By Remark 4.17 again, $\sigma_{i}\left(\theta^{\prime}\right) \geq \sigma_{i}^{0}$.

Case $7 \theta_{i}=\theta_{m}<\theta_{i}^{\prime}$.

The analysis of this case follows from Case 6 and is omitted.

This completes the proof of strategyproofness. For $(n-1) / 2$-queue inefficiency, let $\theta$ be a profile such that $\theta_{i} \neq \theta_{j}$ for all $i$ and $j, i \neq j$ so that the efficient queue $\sigma^{e}$ is unique. Let $i_{m}$ be the (unique) agent with the median waiting $\operatorname{cost} \theta_{m}$. Observe that $\sigma_{i}^{e}(\theta) \gtreqless(n+1) / 2$ if $\theta_{i} \lesseqgtr \theta_{m}$. We will show that $\left|\sigma_{i}(\theta)-\sigma_{i}^{e}(\theta)\right| \leq(n-1) / 2$ for all $i \in N$.

(1) If $i=i_{m}$, then $\sigma_{i_{m}}^{e}(\theta)=(n+1) / 2$ and $\left|\sigma_{i}(\theta)-\sigma_{i}^{e}(\theta)\right| \leq \max \{[(n+1) / 2]-$ $1, n-[(n+1) / 2]\}=(n-1) / 2$.

(2) Let $\theta_{i}>\theta_{m}$. Assume, for the purpose of contradiction, that $\sigma_{i}(\theta)>(n+1) / 2$. Then, there exists $j^{*} \in N \backslash\left\{i, i_{m}\right\}$ such that $\theta_{j^{*}}<\theta_{m}$ and $\sigma_{j^{*}}(\theta) \leq(n+1) / 2$. If not, $\theta_{k} \geq \theta_{m}$ for all $k$ such that $\sigma_{k}(\theta) \leq(n+1) / 2$ and so, $\left|\left\{j: \theta_{j} \geq \theta_{m}\right\}\right| \geq$ $[(n+1) / 2]+1=(n+3) / 2$. Since $\theta_{i} \neq \theta_{j}$ for all $i \neq j$, this means that $\theta_{m}$ is not the median waiting cost, a contradiction.

Consider the pair $\left\{i, j^{*}\right\}$. Assume this pair is at position $k$ in the order $\succ$. Since $\theta_{i}>\theta_{m}>\theta_{j^{*}}$, Remark 4.17 implies that at each stage of the mechanism $\mu^{m}$, agent $i$ can only move forward and agent $j^{*}$ can only move backward in the queue. Hence, at (the beginning of) stage $k, \sigma_{i}^{k-1}(\theta) \geq \sigma_{i}(\theta)>(n+1) / 2 \geq \sigma_{j^{*}}(\theta) \geq$ $\sigma_{j^{*}}^{k-1}(\theta)$. By Definition 4.13, agents $i$ and $j^{*}$ have a feasible exchange at stage $k$. Hence $\sigma_{i}^{k}(\theta)=\sigma_{j^{*}}^{k-1}(\theta) \leq(n+1) / 2$. By Remark 4.17 again, $\sigma_{i}(\theta) \leq \sigma_{i}^{k}(\theta) \leq$ $(n+1) / 2$, a contradiction of our initial assumption. Hence, $\sigma_{i}(\theta) \leq(n+1) / 2$ and $\left|\sigma_{i}(\theta)-\sigma_{i}^{e}(\theta)\right| \leq[(n+1) / 2]-1=(n-1) / 2$.

(3) If $\theta_{i}<\theta_{m}$, then we can use a similar logic to that used in Case (2) to show that $\sigma_{i}(\theta) \geq(n+1) / 2$ and establish that $\left|\sigma_{i}(\theta)-\sigma_{i}^{e}(\theta)\right| \leq(n-1) / 2$.

This completes the proof of the theorem.

Example 4.20 The bound of $(n-1) / 2$-queue inefficiency may not be satisfied if we require the first condition in Definition 4.7 to hold at all profiles. Let $n=7$. Suppose that the initial queue $\sigma^{0}$ is given by $\sigma_{i}^{0}=i$ and the pair $(1,7)$ is first in the order $\succ$. Consider the profile $\theta$ such that $\theta_{7}>\theta_{6}>\theta_{2}=\theta_{3}=\theta_{4}=\theta_{5}>\theta_{1}$.

The median waiting cost for $\theta$ is $\theta_{2}=\theta_{3}=\theta_{4}=\theta_{5}$. At the first stage of the algorithm, there is a feasible exchange between agents 1 and 7 leading to $\sigma^{1}$ where $\sigma_{1}^{1}=7, \sigma_{2}^{1}=2, \sigma_{3}^{1}=3, \sigma_{4}^{1}=4, \sigma_{5}^{1}=5, \sigma_{6}^{1}=6$, and $\sigma_{7}^{1}=1$. After this, there is no feasible exchange. To see this, note that the median agents cannot be part of any feasible exchange. Agent 7 is already in the first queue position and there can be no feasible exchange involving her. The only remaining possibility is an exchange between agents 1 and 6 but this is not feasible because $\sigma_{6}^{1}=6<\sigma_{1}^{1}=7$. Since agent 6's queue position is 2 in all efficient queues, we have

$$
\forall \sigma^{e} \in E(\theta): \quad\left|\sigma_{6}^{1}-\sigma_{6}^{e}\right|=4>\frac{7-1}{2}=3 .
$$

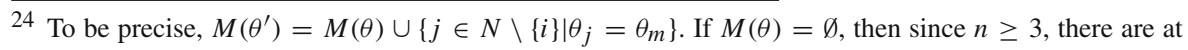
least two agents with waiting $\operatorname{cost} \theta_{m}$ in the profile $\theta$ and therefore, $M\left(\theta^{\prime}\right) \neq \varnothing$.
} 
Remark 4.21 The mechanism $\mu^{m}$ is not strongly group strategyproof because agent $i_{m}$ can change her report and make another agent strictly better-off. Suppose $n=3$, $\sigma_{i}^{0}=i$ for all $i$, and $\theta_{3}>\theta_{2}>\theta_{1}$. Under the mechanism $\mu^{m}$, the only feasible trade is between agents 1 and 3 at the price $\theta_{2}$. However, the coalition $\{2,3\}$ can deviate by changing 2's announcement to $\theta_{1}<\theta_{2}^{\prime}<\theta_{2}$. This leaves agent 2's utility unchanged but agent 3 is better-off because the price she has to pay is now lower. However, the mechanism $\mu^{m}$ is weakly group strategyproof because there is no deviation for a group making all deviating agents strictly better-off. ${ }^{25}$

Remark 4.22 When there is no initial queue, Chun et al. (2014a, 2016) and Kayi and Ramaekers (2010) show that there are mechanisms satisfying OE, BB, SP and different notions of fairness. A natural question that now arises is whether the mechanism $\mu^{m}$ satisfies any fairness properties. This is not easy to answer because it is not clear what fairness means in our context. When there is no initial queue, equal treatment of equals (see Remark 2.12) is a natural fairness requirement. However, when there is an initial queue, every agent is different because no two agents have the same initial queue position.

Curiel et al. (1989) look at the same problem as ours except that they get rid of the mechanism design problem (by assuming that the waiting costs are known publicly) and focus on the "fair sharing of waiting costs." To understand their fairness criteria, observe that one can move from an (inefficient) queue to an efficient queue by sequentially interchanging the positions of two agents who occupy consecutive queue positions. Fairness, according to Curiel et al., requires that every time two agents exchange positions, the resulting saving in waiting cost should be shared equally between the two agents involved in the exchange.

While this fairness criterion is a reasonable one, it will not be satisfied by our mechanism. For instance, suppose $n=3, \sigma_{i}^{0}=i$ and $\theta_{3}>\theta_{2}>\theta_{1}$. Then, the only feasible trade is between agents 1 and 3 who trade at the price of $\theta_{2}$. Agent 1 's gain from the trade is $2 \theta_{2}-2 \theta_{1}$ but agent 3 's gain is $2 \theta_{3}-2 \theta_{2}$ and the two gains are not necessarily equal.

\subsection{Even number of agents}

If the number of agents is even, we face a difficulty because the median waiting cost is typically not well-defined. We can deal with this difficulty in one of two ways. Firstly, as in Moulin (1980), we can introduce phantom agents. Alternatively, we can choose an agent $a$ priori, keep her queue position fixed (at the initial queue position) and apply the mechanism $\mu^{m}$ to the remaining set of $n-1$ agents. Note that both methods involve a modification so that the modified problem effectively involves an odd number of agents.

In the second method, queue inefficiency is minimized by choosing the agent whose queue position in the initial queue is $n / 2{ }^{26}$ To be precise, let $i_{0}^{*}$ be the agent such that

\footnotetext{
25 The argument is similar to the one used to show that the median voter mechanism with single-peaked preferences is weakly group strategyproof.

26 One could, of course, choose the the agent in the queue position $(n / 2)+1$ instead.
} 
$\sigma_{i_{0}^{*}}^{0}=n / 2$. Let $i_{m}^{*}$ be the agent with the median waiting cost in the profile $\theta_{-i_{0}^{*}}$. The mechanism $\mu_{E}^{m}$ redefines the median waiting cost as $\theta_{i_{m}^{*}}$ and modifies the first rule of the mechanism $\mu^{m}$ (see Definition 4.15) as follows:

$\left(1^{\prime}\right)$ If there is no feasible exchange between $i_{k}$ and $j_{k}$, or $i_{0}^{*} \in\left\{i_{k}, j_{k}\right\}$, then $\sigma^{k}=\sigma^{k-1}$ and $t^{k}=t^{k-1}$. Move to stage $k+1$.

Remark 4.23 We can think about the mechanism $\mu_{E}^{m}$ in the following way. First, remove agent $i_{0}^{*}$ from the queue, so that the initial queue is now $\sigma^{0,-i_{0}^{*}}$ [see (2.3)]. We now have the profile $\theta_{-i_{0}^{*}}$ with an odd number of agents, and so we can implement the mechanism $\mu^{m}$. This gives us an allocation $\left(\sigma\left(\theta_{-i_{0}^{*}}\right), t\left(\theta_{-i_{0}^{*}}\right)\right)$.

We now reintroduce agent $i_{0}^{*}$ back into the queue at the position $n / 2$ and set $(\sigma(\theta), t(\theta))$ as follows:

$$
\sigma_{i}(\theta)= \begin{cases}n / 2 & \text { if } i=i_{0}^{*}, \\ \sigma_{i}\left(\theta_{-i_{0}^{*}}\right) & \text { if } i \neq i_{0}^{*} \text { and } \sigma_{i}\left(\theta_{-i_{0}^{*}}\right)<n / 2 \\ \sigma_{i}\left(\theta_{-i_{0}^{*}}\right)+1 \text { otherwise. }\end{cases}
$$

The transfers now are

$$
t_{i}(\theta)= \begin{cases}0 & \text { if } i=i_{0}^{*} \\ t_{i}\left(\theta_{-i_{0}^{*}}\right)-\theta_{i_{m}^{*}} & \text { if } i \neq i_{0}^{*} \text { and } \sigma_{i}(\theta)<n / 2<\sigma_{i}^{0}, \\ t_{i}\left(\theta_{-i_{0}^{*}}\right)+\theta_{i_{m}^{*}} & \text { if } i \neq i_{0}^{*} \text { and } \sigma_{i}^{0}<n / 2<\sigma_{i}(\theta) \\ t_{i}\left(\theta_{-i_{0}^{*}}\right) & \text { otherwise. }\end{cases}
$$

Hence, the reintroduction of $i_{0}^{*}$ causes all agents at positions $n / 2$ or more to move back one position. An agent receives or pays an additional transfer of $\theta_{i_{m}^{*}}$ if her final and initial queue positions are on "different sides" of the position $n / 2$.

Example 4.24 Suppose $\sigma^{0}=$ (4123) meaning that agent 4 initially is in the first position, agent 1 in the second position and so on. Let $\theta_{1}>\theta_{2}>\theta_{3}>\theta_{4}$. Removing agent 1 gives the queue (423) involving the agents $\{2,3,4\}$. Applying $\mu^{m}$ to this set of agents gives the queue (243). The corresponding transfers are $t_{2}\left(\theta_{-1}\right)=-\theta_{3}=-t_{4}\left(\theta_{-1}\right), t_{3}\left(\theta_{-1}\right)=0$. Reintroducing agent 1 gives the queue (2143). Since $\sigma_{2}^{0}=3>2>1=\sigma_{2}(\theta)$, agent 2 has to make an additional payment of $\theta_{3}$; correspondingly, agent 4 receives an additional payment of $\theta_{3}$. Hence, we have $t_{1}(\theta)=t_{3}(\theta)=0, t_{2}(\theta)=-2 \theta_{3}=-t_{4}(\theta)$.

Remark 4.25 Let $\theta$ be a profile such that $\theta_{i} \neq \theta_{j}$ if $i \neq j$. Let $I=\left\{i \neq i_{0}^{*}, i_{m}^{*} \mid \theta_{i}>\right.$ $\left.\theta_{i_{m}^{*}}\right\}, J=\left\{i \neq i_{0}^{*}, i_{m}^{*} \mid \theta_{i}<\theta_{i_{m}^{*}}\right\}$. Remove agent $i_{0}^{*}$ from the economy and apply the mechanism $\mu^{m}$ to the profile $\theta_{-i_{0}^{*}}$. Since the efficient queue for the profile $\theta_{-i_{0}^{*}}$ is unique, Theorem 4.19 implies that $\sigma_{i}\left(\theta_{-i_{0}^{*}}\right) \leq n / 2$ for $i \in I$ and $\sigma_{i}\left(\theta_{-i_{0}^{*}}\right) \geq n / 2$ for $i \in J$. Reintroduce agent $i_{0}^{*}$ back into the economy. By Remark 4.23, it now follows that $\sigma_{i}(\theta) \leq(n / 2)+1$ if $i \in I$ and $\sigma_{i}(\theta) \geq(n / 2)+1$ if $i \in J$. 
Corollary 4.26 Let $n \geq 4$ and even. The mechanism $\mu_{E}^{m}$ satisfies BB, SP, IR and is at most $n / 2$-queue inefficient.

Proof The mechanism $\mu_{E}^{m}$ satisfies BB, SP and IR because the mechanism $\mu^{m}$ has these properties. To show $n / 2$-queue inefficiency, let $\theta$ be a profile such that the efficient queue $\sigma^{e}(\theta)$ is unique.

(1) If $i=i_{0}^{*}$, then $\sigma_{i}(\theta)=\sigma_{i}^{0}=n / 2$ and $\left|\sigma_{i}(\theta)-\sigma_{i}^{e}(\theta)\right| \leq \max \{n-(n / 2),(n / 2)-$ $1\}=n / 2$.

(2) If $i=i_{m}^{*}$, then $\sigma_{i}(\theta)=\sigma_{i}^{0}$. Depending on whether $\theta_{i_{0}}<\theta_{i_{m}^{*}}$ or $\theta_{i_{0}}>\theta_{i_{m}^{*}}, \sigma_{i}^{e}(\theta) \in$ $\{n / 2,(n / 2)+1\}$. Hence, $\left|\sigma_{i}(\theta)-\sigma_{i}^{e}(\theta)\right| \leq \max \{(n / 2)+1-1, n-(n / 2)\}=n / 2$.

(3) Let $i \neq i_{0}^{*}, i_{m}^{*}$. Then, either $i \in I$ or $i \in J$. By Remark 4.25, $\sigma_{i}(\theta) \leq(n / 2)+1$ for $i \in I$ and $\sigma_{i}(\theta) \geq(n / 2)+1$ for $i \in J$. To find maximal queue inefficiency, we need to work out bounds on $\sigma_{i}^{e}(\theta)$. We have already noted that $\sigma_{i_{m}^{*}}^{e}(\theta) \in$ $\{n / 2,(n / 2)+1\}$. It follows from this that $\sigma_{i}^{e}(\theta) \leq \max \{(n / 2)-1, n / 2\}=n / 2$ for all $i \in I$ and $\sigma_{i}^{e}(\theta) \geq \min \{(n / 2)+1,(n / 2)+2\}=(n / 2)+1$ for all $i \in J$. Hence, for all $i \in I,\left|\sigma_{i}(\theta)-\sigma_{i}^{e}(\theta)\right| \leq[(n / 2)+1]-1=n / 2$. Similarly, for all $i \in J,\left|\sigma_{i}(\theta)-\sigma_{i}^{e}(\theta)\right| \leq|[(n / 2)+1]-n| \leq n / 2$.

\section{Conclusion}

The central message of this paper is that the presence of an initial queue in the queueing problem (single server, identical processing times) leads to very different results. Budget balance, strategyproofness, queue efficiency, and identical preferences lower bound are compatible in the model without an initial queue but their counterparts are not compatible if there is an initial queue. One way to understand the difference is that the model without an initial queue corresponds to a pure public good model but the presence of an initial queue adds a private good aspect as well. This, not surprisingly, leads to incompatibility. Since the four desirable properties are incompatible, one or more of the properties must be relaxed. In this paper we have examined the consequences of relaxing budget balance and outcome efficiency. The results overall are negative.

Finally, as a topic for future research, this model can be extended in many directions. One can consider multiple identical or non-identical facilities, or extend the analysis by dropping the identical processing time assumption. All these extensions certainly have real life applications and hence are worth pursuing.

Open Access This article is distributed under the terms of the Creative Commons Attribution 4.0 International License (http://creativecommons.org/licenses/by/4.0/), which permits unrestricted use, distribution, and reproduction in any medium, provided you give appropriate credit to the original author(s) and the source, provide a link to the Creative Commons license, and indicate if changes were made.

\section{References}

Barberà S, Jackson M (1995) Strategy-proof exchange. Econometrica 63:51-87

Chun Y (2006) No-envy in queueing problems. Econ Theory 29:151-162 
Chun Y (2006) A pessimistic approach to the queueing problem. Math Soc Sci 51:171-181

Chun Y, Mitra M, Mutuswami S (2014) Egalitarian equivalence and strategyproofness in the queueing problem. Econ Theory 56(2):425-442

Chun Y, Mitra M, Mutuswami S (2014) Characterizations of pivotal mechanisms in the queueing problem. Math Soc Sci 72(1):62-66

Chun Y, Mitra M, Mutuswami S (2016) A characterization of the symmetrically balanced VCG mechanism in the queueing problem. Games Econ Behav (forthcoming)

Clarke EH (1971) Multi-part pricing of public goods. Public Choice 11:17-33

Cramton P, Gibbons R, Klemperer P (1987) Dissolving a partnership efficiently. Econometrica 55(3):615632

Curiel I, Pederzoli G, Tijs S (1989) Sequencing games. Eur J Oper Res 40:344-351

Gale D, Shapley LS (1962) College admissions and the stability of marriage. Am Math Monthly 69:9-15

Gershkov A, Schweinzer P (2010) When queueing is better than push and shove. Int J Game Theory 39:409-430

Goswami MP, Mitra M, Sen A (2014) Strategy-proofness and Pareto-efficiency in quasi-linear exchange economies. Theor Econ 9(2):361-381

Groves T (1973) Incentives in teams. Econometrica 41:617-631

Hagerty K, Rogerson W (1987) Robust trading mechanisms. J Econ Theory 42:94-107

Holmström B (1979) Groves' schemes on restricted domains. Econometrica 47:1137-1144

Kayi C, Ramaekers E (2010) Characterizations of Pareto-efficient, fair, and strategy-proof allocation rules in queueing problems. Games Econ Behav 68:220-232

Maniquet $\mathrm{F}$ (2003) A characterization of the Shapley value in queueing problems. J Econ Theory 109:90-103

Mitra M (2001) Mechanism design in queueing problems. Econ Theory 17:277-305

Mitra M, Mutuswami S (2011) Group strategyproofness in queueing models. Games Econ Behav 72:242254

Moulin H (1980) On strategy-proofness and single peakedness. Public Choice 35:437-455

Roth AE (1982) Incentive compatibility in a market with indivisible goods. Econ Lett 9:127-132

Schummer J, Abizada A (2016) Incentives in landing slot problems. J Econ Theor (forthcoming)

Schummer J, Vohra RV (2013) Assignment of arrival slots. Am Econ J Microecon 5(2):164-185

Shapley LS, Scarf H (1974) On cores and indivisibility. J Math Econ 1:23-38

Suijs J (1996) On incentive compatibility and budget balancedness in public decision making. Econ Des 2:193-209

Vickrey W (1961) Counterspeculation, auctions and competitive sealed tenders. J Finance 16:8-37 